\title{
Research on the Electric Vehicle Control System
}

\author{
Haiying Wang, Tianjun Sun, Xingbo Zhou and Qi Fan \\ School of Automation, Harbin University of Science and Technology, Harbin, \\ China \\ E-mail:wanghy@hrbust.edu.cn
}

\begin{abstract}
With the development of electric vehicle electronic control unit (ECU) for vehicle control technology and the key to raise the level of design electric vehicles the optimization control of the vehicle performance as direction. In order to achieve the rational, coordination of vehicles within the system of integrated control, based on the modular thought, through constructing the distributed control network design overall structure of the control system of pure electric vehicle, the vehicle controller is analyzed on the working principle and its function realization degree, as a pure electric vehicle control system provides the theory basis for performance evaluation.
\end{abstract}

Keywords: Electric vehicles, Vehicle control system, Vehicle controller

\section{Introduction}

Modern electric car research and development is based on the theory of modern control technologies, including automotive technology, motor driver technology, power electronics technology and energy storage technology, etc.

The design process of the electric car three major problems need to be solved, are respectively the vehicle technology, electric drive technology and energy management system. Among them, the vehicle technology includes two aspects: one is the vehicle body technology, mainly by reducing electric car body weight, to realize the acceleration and grade ability and effectively increase the limited driving distance; Secondly, the vehicle control system integrated control technology, mainly for reasonable arrangement and coordination of the electronic control unit (ECU) for electric vehicles, save material and reduce cost, realize the system optimization of multiple factors in the process of optimization, improve the vehicle performance [1].

In traditional fuel vehicle, the engine management system (EMS) to make car engine in all under the condition of low pollutant emission control, improve fuel economy and excellent driving performance; For pure electric vehicles, it shall replace the original motor engine, with power battery instead of fuel oil ACTS as a source of power, therefore, pure electric vehicles also need a travel distance to increase its vehicle management system, optimizing the allocation of energy. And the vehicle control system has a significant influence on vehicle driving performance, is the important content of pure electric vehicle control technology research [2].

\section{The Analysis of System Design Requirements}

At home, the research of pure electric vehicle control technology is more preference for engineering application; Abroad, the research towards more braking energy recycling, of the electronic control unit (ECU) for the traction control and stability control.

Because of pure electric vehicle control system integration of electrical, electronic, mechanical, chemical and other nonlinear dynamic system, complicated structure, which includes motor, converters, power battery, transmission, and other electric control 
equipment, so, no matter how electric cars other assembly performance in good condition, once there was a problem in the vehicle controller, electric vehicles will not be able to run normally, cannot achieve energy recovery control, or even an accident. On how to coordinate all parts of function units, make it reasonable and efficient for work of pure electric vehicle controller is a very thorny problem in the design process?

Based on the research status of the vehicle control system at home and abroad, in view of some problems in the process of vehicle control technology, the paper builds the vehicle control system overall frame, the vehicle controller is analyzed on the working principle, ultimately determine its functions.

\section{The Construction of Vehicle Control Network}

Vehicle Controller as a pure Electric Vehicle Control Unit, through the electronic Control Unit to collect real-time dynamic parameters of the controlled object and feedback, and then through the CAN bus is passed to the core Controller for operation analysis, and then by the VCU to ECU Control instruction, make the reasonable coordination of efficient performance of ECU, realize the Control of the Vehicle drive performance and energy management, optimization, to ensure the Electric performance.

In general, the realization of the function of the pure electric vehicle controller scheme has two kinds: centralized control and distributed control, as shown in Table 1.

Table 1. The Realization of the Function of Electric Vehicle Controller Scheme

\begin{tabular}{l|c|c|c}
\hline & control method & advantages & disadvantages \\
\hline Centralized control & $\begin{array}{c}\text { By the core } \\
\text { processor to } \\
\text { complete the } \\
\text { processing of signal, } \\
\text { energy data and } \\
\text { distribution of work }\end{array}$ & $\begin{array}{c}\text { deal with } \\
\text { concentration } \\
\text { high implement } \\
\text { fast response } \\
\text { low cost }\end{array}$ & $\begin{array}{c}\text { PCB complex } \\
\text { bad heat dissipation }\end{array}$ \\
\hline $\begin{array}{c}\text { By the core } \\
\text { controller through } \\
\text { field bus and } \\
\text { communication } \\
\text { electronic control } \\
\text { unit (ECU) for each }\end{array}$ & $\begin{array}{c}\text { modular } \\
\text { low complexity } \\
\text { flexible } \\
\text { configuration }\end{array}$ & complicated structure \\
\hline
\end{tabular}

Because the CAN bus car traffic more and more used in distributed control system, and combining the Table 1 provided two kinds of control scheme of the analysis, this study adopts the distributed control system based on CAN bus, in the process of pure electric vehicle controller control, for the ECU by CAN bus communication and control.

Distributed control system based on modular construction; improve the correlation between each subsystem of the vehicle. The system can be divided into two layers: the whole car controller and the secondary controller. As shown in Figure 1, among them, the whole car controller (VCU) as the top processing information and publish the instructions, the secondary controller in the Motor controller vehicle combination Instrument and Battery Management System as the subsystem of the independent Management and monitoring of the respective Control object. Real-time state parameter subsystem at all levels will be collected by CAN bus to realize sharing information exchange to $\mathrm{VCU}$, then by $\mathrm{VCU}$ information comprehensive, at all levels to implement the controller between the centralized and decentralized control, information interaction function [3-4]. 


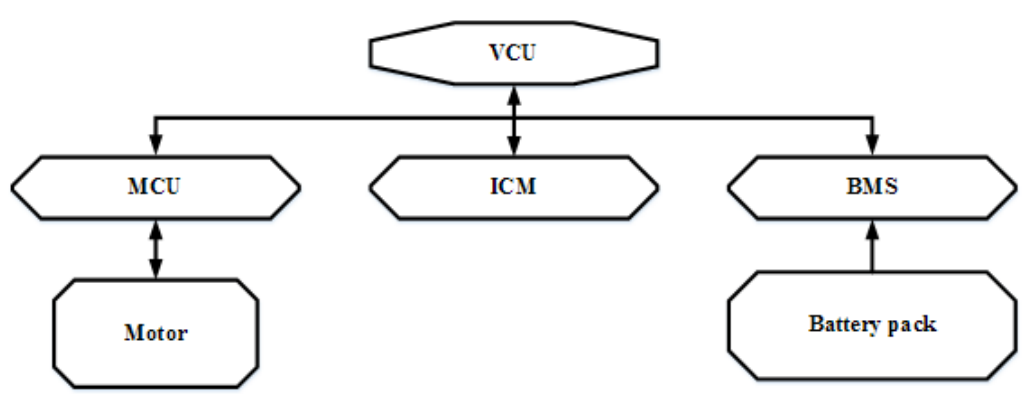

Figure 1. Hierarchical Control System Schematic Diagram of the Vehicle

\section{The Summary of the Electric Vehicle Control System}

As shown in Figure 2, pure electric vehicle dynamic control system can be divided into three parts, respectively for the vehicle control system, motor drive system and power supply system. The core of the whole vehicle control system is vehicle control technology, the technology including vehicle structure design of control software, control strategy and energy management. At the same time, the vehicle controller as an objectively reflect the actual development of single chip micro control system for vehicle control technology, electronic control unit as a measure of the core component of the vehicle control system performance and function level, its function realization degree directly affect the control effect of the control system of the vehicle, has become one of the evaluation criteria of vehicle controller performance is good [5].

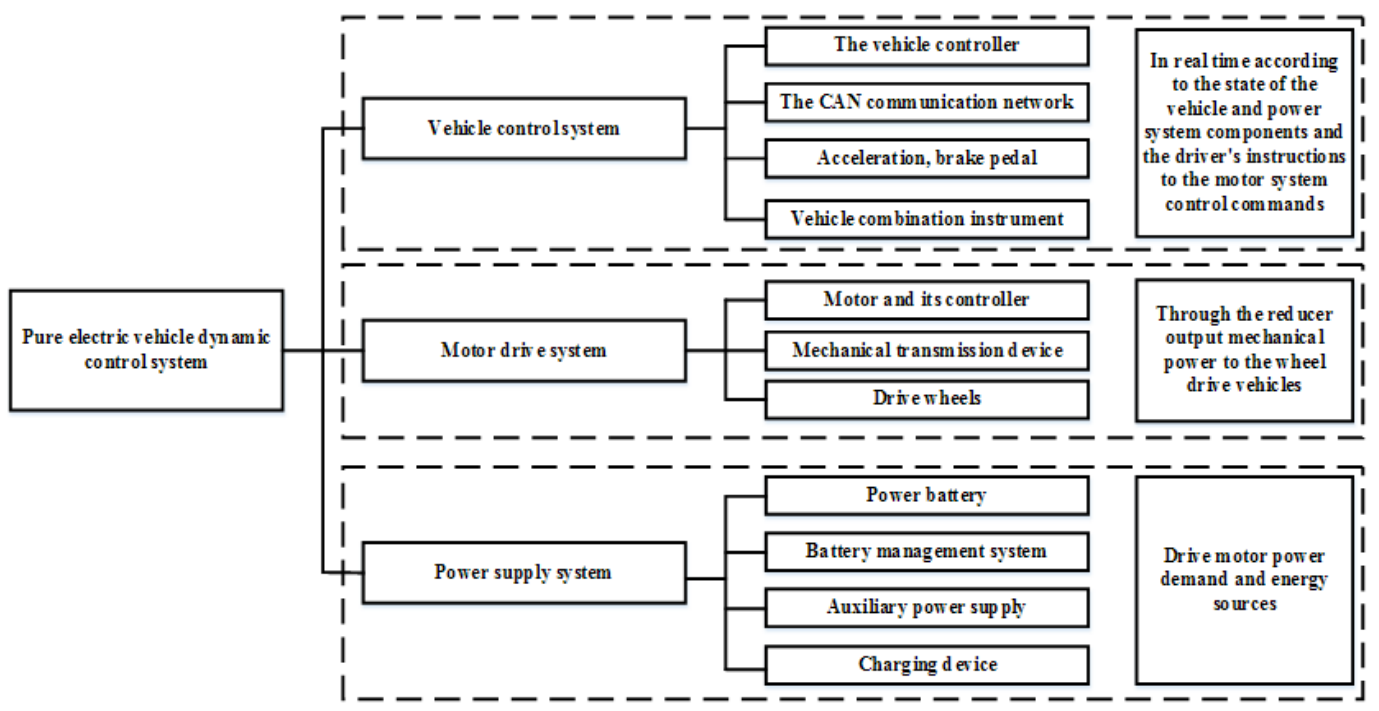

Figure 2. Pure Electric Vehicle Dynamic Control System Structure

Vehicle controller as a pure electric vehicle control system is the most important core part, is responsible for the overall control, coordination and monitoring vehicle running state, the operation of the pilot signal and the signal from the sensor via the control strategy, inherent to the vehicle controller to the electronic control unit, energy management, fault diagnosis and the vehicle running state control, monitoring, and other functions, and through the LCD automotive instrument display. Therefore, to develop fully functional vehicle controller, improve the design of the control system of electric vehicle. The vehicle controller diagram is shown in Figure 3 [6-8]. 


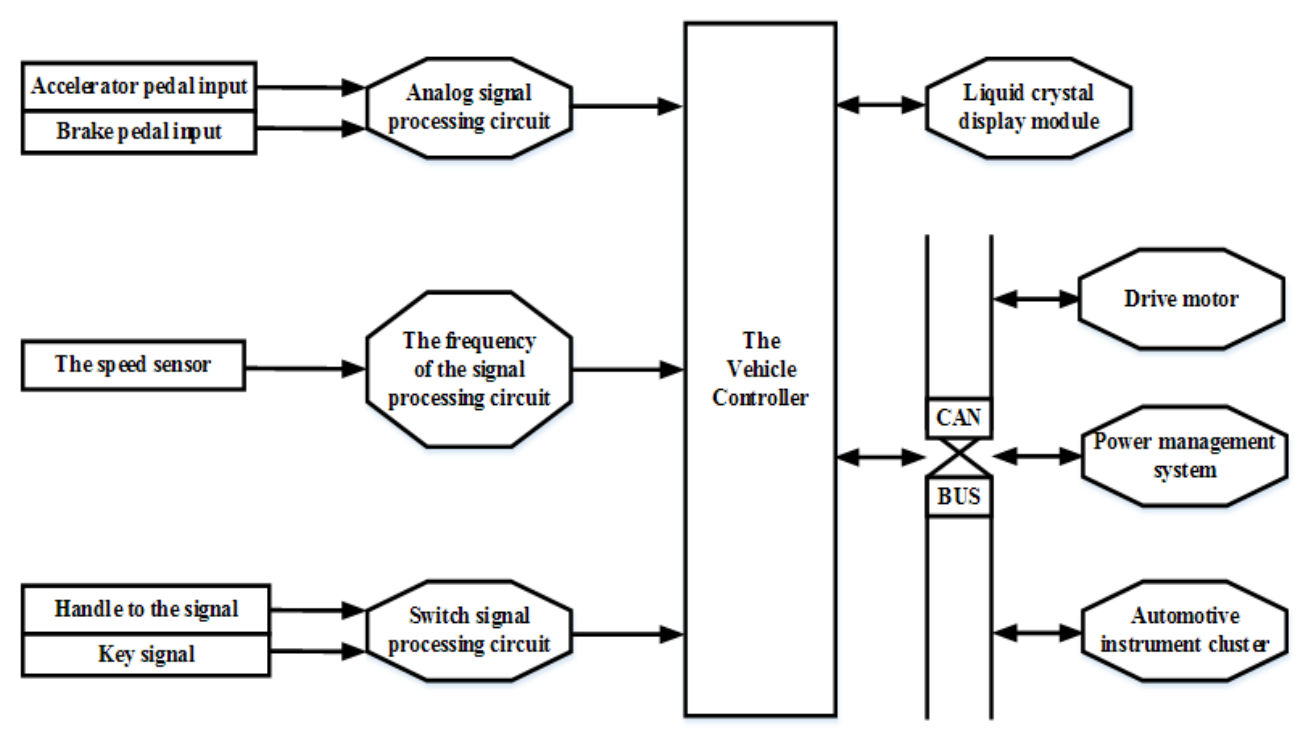

Figure 3. Vehicle Controller

\section{Working Principle of the Control System for Vehicle}

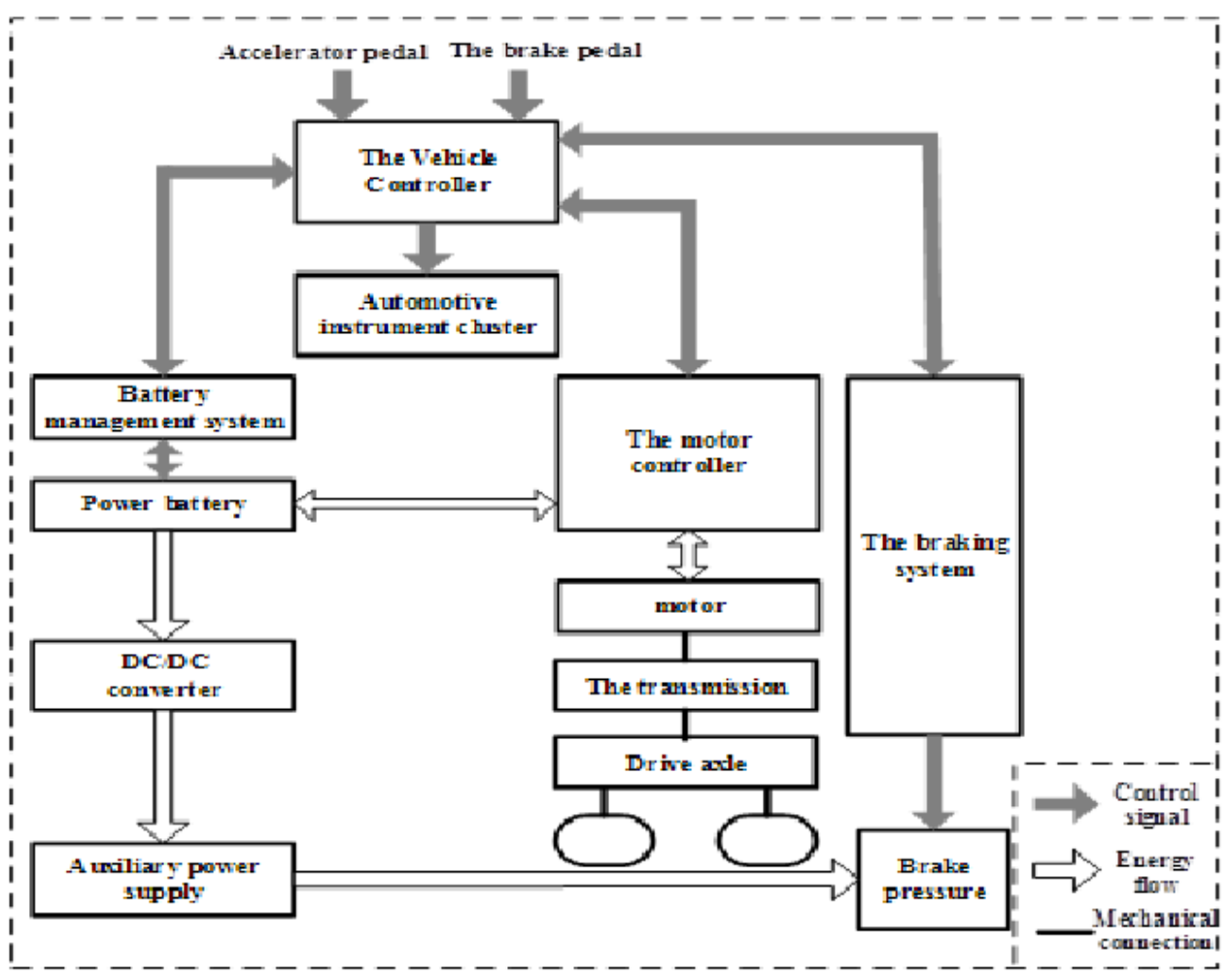

Figure 4. Vehicle Controller Works

The vehicle control system is a closed-loop control system, artificial according to perform a task hierarchy can be divided into three layers: policy makers, formed by the driver itself; Coordination layer, based on the vehicle controller processing real-time status information and control instructions for the operation attempt of driver is reasonable judgment; Executive level, handle relevant control instruction made by vehicle controller. In order to improve the safety of the vehicle control system stability, rely on 
the system control error feedback correction. Working principle of the whole vehicle controller is shown in Figure 4.

Pure electric vehicle charging is accomplished by charging equipment on the ground, when the driver use the key to start switch, power battery to the signal through the DC/DC converter, instrument and low voltage electrical equipment such as power supply for lighting. At the same time, through the vehicle controller also supply power to the motor controller, dc inverter motor controller as three-phase alternating current for the motor running, and then through the transmission, the rotation axis, differential and rear axle drive vehicle. The vehicle controller can be realized by controlling the motor of the vehicle to realize forward or backward.

When the vehicle is started, the driver will first block a choice in the "forward" or "backward" position, and then stepped on the accelerator pedal () on the accelerator. When the accelerator pedal off after the initial position, the driver can be done by changing the accelerator pedal opening of driving torque given value change, namely when the accelerator pedal opening (exactly) the accelerator, the largest drive torque given value most; When the opening of the accelerator pedal is minimum, throttle back in situ, drive torque given minimum value. When the motor controller receives the control signals and drive torque given value, the energy flow to the motor, the control of its operation, and according to the control signal to determine motor rotation direction, at the same time, according to the given value driving torque signal to determine the magnitude of torque.

Pure electric vehicle braking mode is divided into two kinds: emergency braking and emergency braking. The former generally rely on mechanical braking system for brake; The latter usually by mechanical braking and regenerative braking of motor brake. According to the scene environment condition, when need to use smaller braking intensity, the braking system will be given priority to with regenerative braking, mechanical brake is complementary; When with the secondary braking intensity, the braking system will be given priority to with mechanical braking, regenerative braking is complementary.

When the driver on the brake pedal brake (start), brake pedal deviates from the initial position, motor controller will combine control signals, brake level and braking torque values given three indicators to control the power of the motor brake state, and based on the brake torque values given signal to determine the size of the motor output brake torque, at the same time open the regenerative braking, to power the battery. When lift the brake pedal brake (cancelled), braking state signal disappears, the braking torque given value also recover (0), then the output of the motor controller to stop brake torque. When vehicles run on deceleration mode, the motor ACTS as a generator converts kinetic energy into mechanical energy to power the battery in order to realize the trip range of pure electric vehicles increased [9].

\section{The Main Function of Electric Vehicle Control System}

Vehicle controller as the core of pure electric vehicles controllers, operation and management of the vehicle and each relevant ECU control plays a vital role. As a result of the different control function of the electronic control unit for each part, the original complex control system can be decomposed into relatively simple single subsystem, and then based on the modular thought clear a small part of each module can realize the function.

On the one hand, it received by the sensor transmits data and instructions by the driver's psychological intentions, and in accordance with the control strategy for processing and analysis, then send control commands to the motor control unit, battery management system and the car instrument cluster control unit, at the same time, the vehicle running status real-time monitoring; On the other hand, in the process of pure electric vehicle brake control of braking energy feedback, and then improve the travel 
distance of pure electric vehicles. Electronic control unit of vehicle controller use CAN bus and other data information to transfer the control instructions. Vehicle controller is regarded as the center of the vehicle communication network nodes, to improve data transmission rate and improve the reliability.

The vehicle controller to realize the main functions are:

(1) Drive torque control

Driving moment of the control, as the most basic, the most important function of the whole vehicle controller, the pedal through gathering information, and then parse the pilot operation intention, to realize the transformation of the demand for motor output torque. In this process, the driver by manipulating the accelerator or the brake pedal to realize signal input, also is the purpose of the will be the driver's operation in the form of a signal transmitted to the vehicle controller, then through analysis after processing by motor vehicle controller according to the output driving moment or braking torque drivers psychological intentions.

(2) The braking energy optimization control

Compared to pure electric vehicles and the traditional energy vehicles, the main difference is that one of the braking energy recovery. Motor as the only power output and has function of motor and generator two - when the driver steps on the gas pedal drive vehicles, motor function is equivalent to the motor; On the contrary, when the driver on the brake pedal to slow down when driving, the function of the motor is equivalent to the generator, also is to take advantage of the electric car braking energy power generation and storage to the energy storage device, when meet certain conditions, and the energy to the battery pack. In this process, the vehicle controller based on the accelerator and brake pedal opening and the current speed and power battery charged State (State of Charge, SOC) to determine whether braking energy recovery at a certain moment, if you can, the vehicle controller issue related to the motor controller instructions, recycling part of the braking energy, improve energy utilization efficiency.

(3) The vehicle energy management

In the process of pure electric vehicles, electric supply system as the only power supply unit in addition to the drive system power supply, but also to similar to the motor, air conditioning and other automotive electronic control unit power supply. In order to extend the range of pure electric vehicle, the vehicle controller can to reasonable optimization of energy, when the battery power is low, the vehicle controller will send off part of the supplementary electrical equipment control instruction, so as to ensure the safety of electricity used in vehicles, thus improving the utilization ratio of energy,

(4) CAN the maintenance and management of the network

Vehicle controller as the master node of communication network, also manages the vehicle network, the communication network based on CAN bus technology, has a number of master-slave node, real-time vehicle network state regulation, and has the priority of information dynamic allocation.

(5) Fault diagnosis and processing

Vehicle controller can continuous real-time monitoring of vehicle running status, including the parts EUD working condition, once the malfunction of the electronic control unit first timely alarm, security measures at the same time, then the fault diagnosis, store and send the error code, provide the basis for the future maintenance and routine inspection car, in the end to the problem of fault classification processing, and for those who do not influence the operation of the vehicle glitches, to maintain the vehicle's moving.

(6) The vehicle condition monitoring

Vehicle controller based on on-board instrumentation control to display the real-time operation state of the vehicle information, to make drivers know vehicle state. Vehicle condition monitoring technology based on CAN bus communication for motor speed, speed, battery remaining power, running state parameters such as current, and the above data through the car instrument cluster intuitive display, improved the car interactivity 
between drivers and vehicles, at the same time as the driver accurately grasp the overall vehicle operation condition to provide convenient conditions [10].

\section{Conclusion}

Pure electric vehicle controller as the core component of the vehicle control system, the moving car, driving moment control, optimization and control of braking energy, the energy of the vehicle management, the CAN network maintenance and management, fault diagnosis and treatment, the vehicle condition monitoring functions such as the implementation of the degree plays a key role.

This paper firstly introduces the composition of pure electric vehicle control network, and using distributed control system is determined, and then through the vehicle controller schematic overview of its system structure, and then describes in detail the working principle of the vehicle controller, finally put forward the vehicle controller can realize the function, as to provide theoretical basis for the further research and development and performance evaluation.

\section{Acknowledgements}

This paper is partially supported by Technological Innovation Foundation for Leaders of Disciplines in Science of Harbin (2014RFXXJ032).

\section{References}

[1] Q. Chen, F. Sun and J. Zhu, "Modern electric vehicle technology", Beijing Institute of Technology press, (2002), pp. 1-3.

[2] Y. Zhang, "Pure electric vehicle powertrain control system research", Doctoral Dissertation of Shanghai Jiao Tong University, vol. 1-3, (2008), pp. 42-45.

[3] L. Wang, "Power of pure electric bus assembly control strategy", Master degree thesis of Jilin University, (2009), pp. 1-2.

[4] L. Wang, "Key technologies and development prospects of electric vehicle", Research on automobile industry, no. 8, (2009), pp. 12-15.

[5] R. Wan, "Study on the strategy of bus scheduling and vehicle control in distributed control system of electric vehicle", Tianjin University doctoral these studies, (2004), pp. 74-77.

[6] J. Zhang, "Controller of pure electric passenger vehicle. Master degree thesis", Jilin: Jilin University, (2008).

[7] Y. Cheng, S. Cui, L. Song and C. C. Chan, "The study of the operation modes and control strategies of an advanced electromechanical converter for automobiles", IEEE Trans. Magn., vol. 43, no. 1, (2007) January, pp. 430-433.

[8] V. H. Johnson, K. B. Wipke and D. J. Rausen, "HEV control strategy for real-time optimization of fuel economy and emissions", SAE Paper, (2000)-01.

[9] Q. Yan, "Hybrid electric vehicle research and development of controller assembly", Huanan: School of mechanical and automotive engineering Hunan University, (2007).

[10] G. Yang, J. Zhang and Y. Liang, "Design of control system of integrated motor controller of electric vehicle", Alternative technology and electric traction, vol. 2, (2007), pp. 39-43. 
International Journal of $u-$ and e- Service, Science and Technology Vol.8, No. 8 (2015) 